Supporting Information

\title{
Correction to NIPAM-based Microgel Microenvironment Regulates Cardiac Stem Cells
}

Xiaolin Cui ${ }^{\mathrm{a} \dagger}$, Junnan Tang, ${ }^{\mathrm{b}, \mathrm{c}, \mathrm{d} \dagger}$, Yusak Hartanto ${ }^{\mathrm{a}}$, Jiabin Zhang ${ }^{\mathrm{a}}$ Jingxiu Bi $^{\mathrm{a}}$, Sheng Dai ${ }^{\mathrm{e}}$, Shi Zhang Qiao ${ }^{\mathrm{a}}$, Ke Cheng, ${ }^{\mathrm{b}, \mathrm{c}, \mathrm{d}^{*}}$ and Hu Zhang ${ }^{\mathrm{a}, \mathrm{f}^{*}}$

a School of Chemical Engineering, The University of Adelaide, Adelaide 5000, Australia.

b Department of Cardiology, The First Affiliated Hospital of Zhengzhou University, Zhengzhou, Henan 450052, China.

c Department of Molecular Biomedical Sciences and Comparative Medicine Institute, North Carolina State University, Raleigh, North Carolina 27607, USA.

d Joint Department of Biomedical Engineering, University of North Carolina at Chapel Hill \& North Carolina State University, Chapel Hill and Raleigh, North Carolina 27599 and 27607, USA.

e School of Chemical Engineering and Advanced Materials, Newcastle University, Newcastleupon-Tyne, NE1 7RU, United Kingdom.

F Amgen Bioprocessing Centre, Keck Graduate Institute, Claremont, California 91711, USA.

*Corresponding authors: Prof. Hu Zhang; Prof. Ke Cheng;

Email: hu.zhang@adelaide.edu.au; kcheng3@ncsu.edu

$\dagger$ Equal contribution 


\section{Materials and Methods}

\section{Materials}

N-Isoproylacrylamide (NIPAM, 99\%+), N, N-methylenebisacrylamide (MBA, 98+), potassium persulfate (KPS, 99\%+), sodium dodecyl sulphate (SDS, 98.5\%+), itaconic acid (IA, 99\%+), 2-hydroxyethyl methacrylate (HEMA), dimethyl amino ethyl methacrylate (DMAEMA), and poly(ethylene glycol) methyl ether (PEGA) were ordered from SigmaAldrich. NIPAM was recrystallized in n-hexane and dried in vacuum at room temperature before use. Collagenase solution was bought from Sigma. Fiberonectin was ordered from Biosciences. Iscove's Modified Dulbecco's Medium (IMDM), 2-mercaptoethanol, Lglutamine, and LIVE/DEAD ${ }^{\circledR}$ viability/cytotoxicity kit were bought from Invitrogen. Fetal bovine serum and ultralow attachment flask were ordered from Corning. Gentanicin and trypsin were purchased from Gibco, Life technologies. VEGF and IGF-1 were purchased from R\&D Systems Mineapolis and the SDF-1 ELISA kits from B-Bridge International in Cupertion.

\section{Microgel characterization}

The hydrodynamic diameters $\left(\mathrm{d}_{\mathrm{h}}\right)$ and zeta potentials of $1 \mathrm{mg} / \mathrm{mL}$ microgels in PBS buffer were measured with a Zetasizer (Malvern, Nano-ZS) at different temperatures. A Thermos Scientific NICOLET 6700 attenuated total reflectance Fourier transform infrared spectrometer (ATRFTIR) was used to confirm the functional groups of the microgels. Dynamic oscillation rheological experiments were performed in a SR5 rheometer (Rheometric Scientific) with a 40 $\mathrm{mm}$ cone plate geometry for $30 \mathrm{mg} / \mathrm{mL}$ microgel dispersions in PBS buffer. The temperature was controlled by a Peltier system, and both elastic (storage) modulus G' and viscous (loss) modulus G" were measured at a temperature range of 20 to $40{ }^{\circ} \mathrm{C}$. All experiments were tested in the linear viscoelastic region.

\section{Hydrogel morphologies}

$30 \mathrm{mg} / \mathrm{mL}$ microgel dispersions in PBS buffer were prepared in centrifuge tubes. The tubes were placed into a water bath at $37{ }^{\circ} \mathrm{C}$ until the solutions became gels. The samples were quickly quenched by liquid nitrogen then dried under vacuum using a Christ Alpha 2-4 LD free dryer. A FEI Quanta 450 FEG Environmental SEM (scanning electron microscopy) was employed to record microgel morphologies after coating with platinum at an acceleration voltage of $20 \mathrm{kV}$. 


\section{Live/dead cell images}

The cell viability test was performed with the Live/Dead cell viability/cytotoxicity kit. After cell culture, the gel /cell mixture was washed with pre-warmed PBS buffer twice. $200 \mu \mathrm{L}$ (for 24 wells) or $50 \mu \mathrm{L}$ (for 96 wells) dye solution ( $0.2 \%$ Ethidium homodimer-1 (red) and $0.05 \%$ calcein AM (green) in PBS buffer) was added into each well. After incubation for another 30 min at $37{ }^{\circ} \mathrm{C}$, cells were rinsed by PBS buffer twice. Cells were observed under a ZEISS LSM 880 WITH Airyscan microscope (Carl Zeiss, Oberkochen, Germany). 


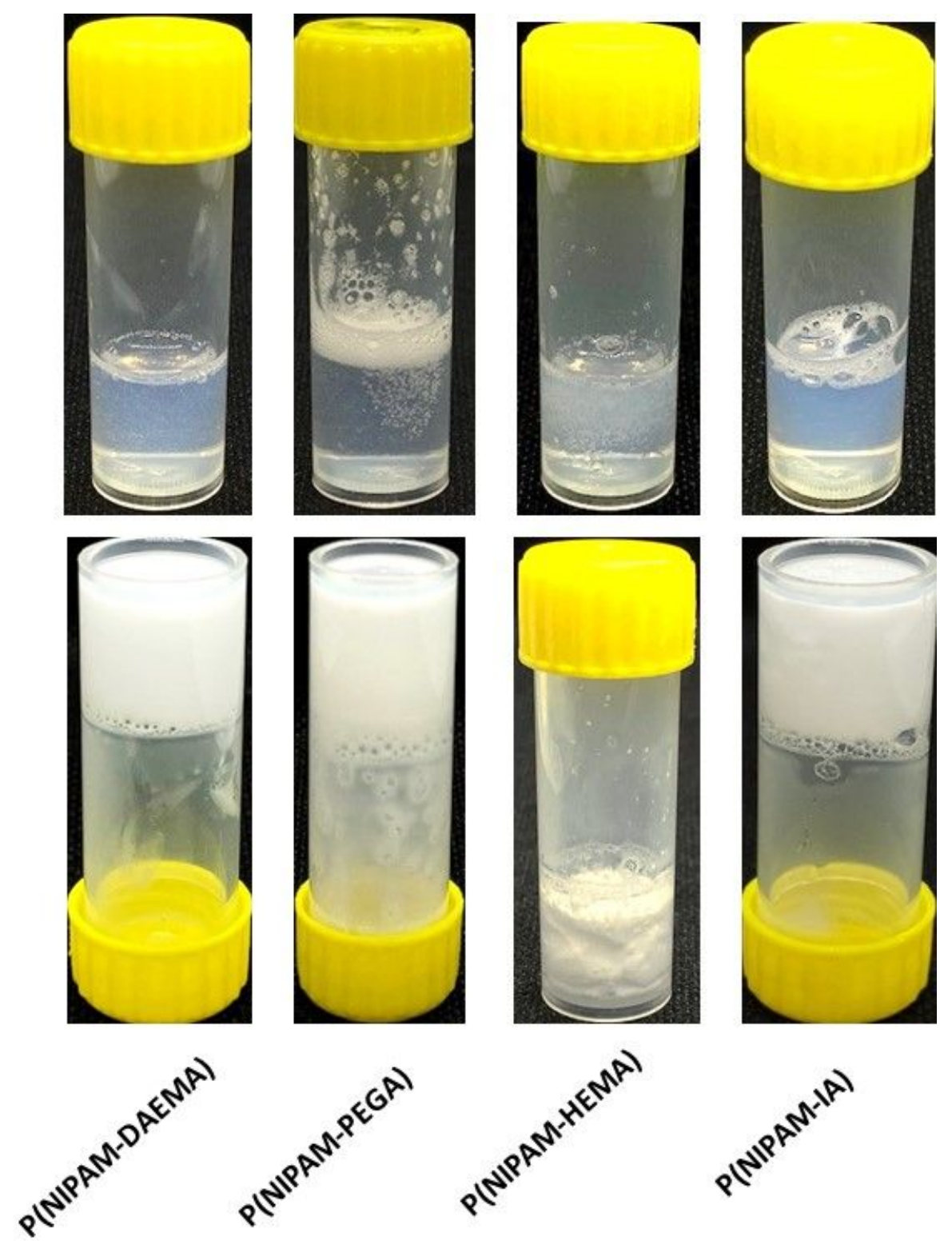

Figure S1. Comparison of $30 \mathrm{mg} \mathrm{mL}^{-1}$ thermo-responsive microgels $(\mathrm{pH} \sim 7.2)$ in their dispersed states $\left(25^{\circ} \mathrm{C}\right)$ and hydrogel states $\left(37^{\circ} \mathrm{C}\right)$. 


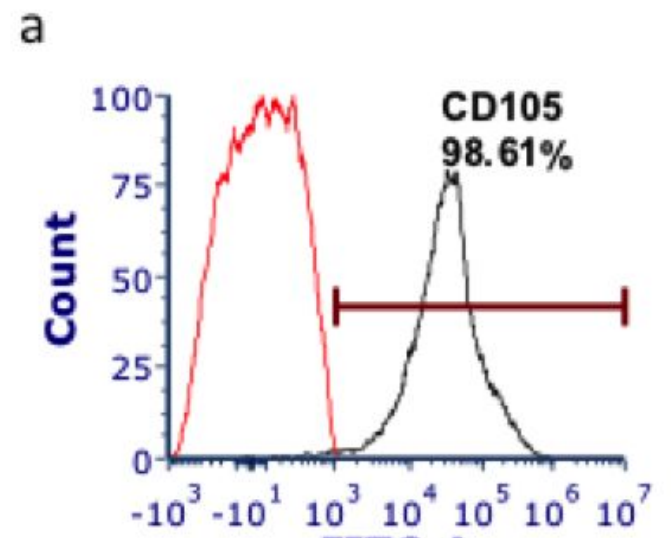

FITC-A

C

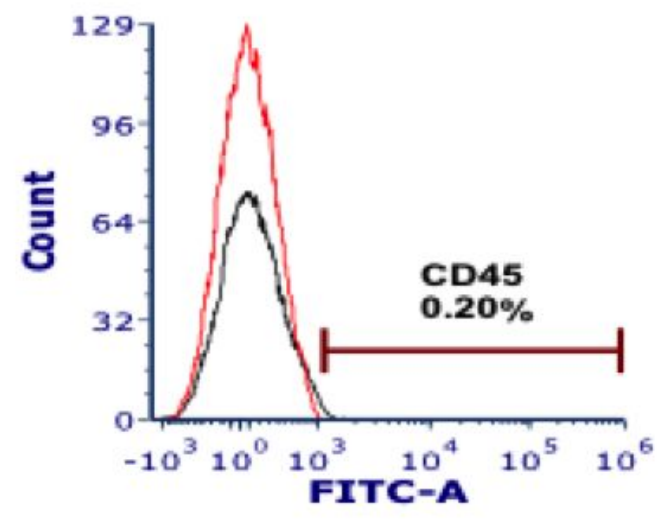

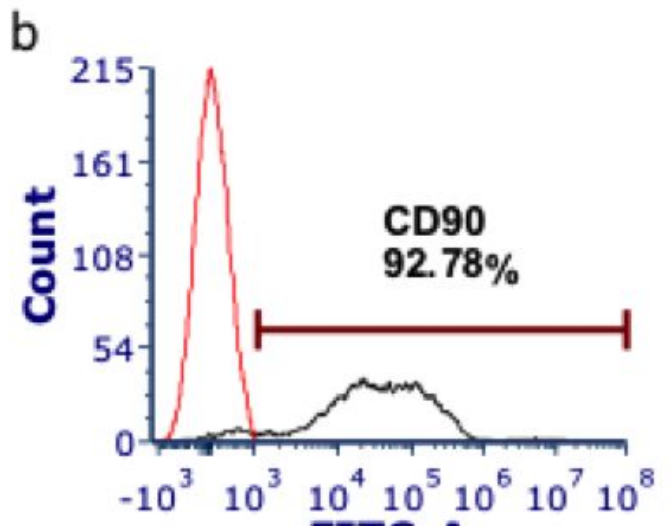

FITC-A

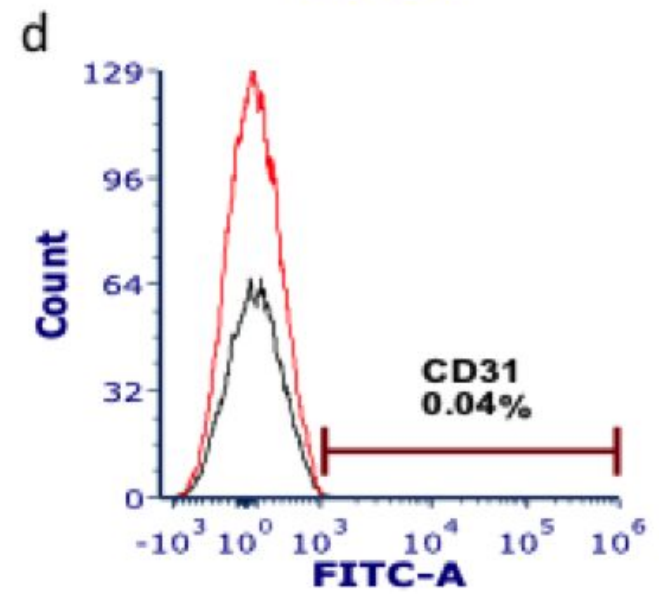

Figure S2. Surface markers for hCSCs determined by flow cytometry. CD105 (a) and CD90 (b) were mainly positive on hCSCs, but not CD45(c) and CD31 (d). 

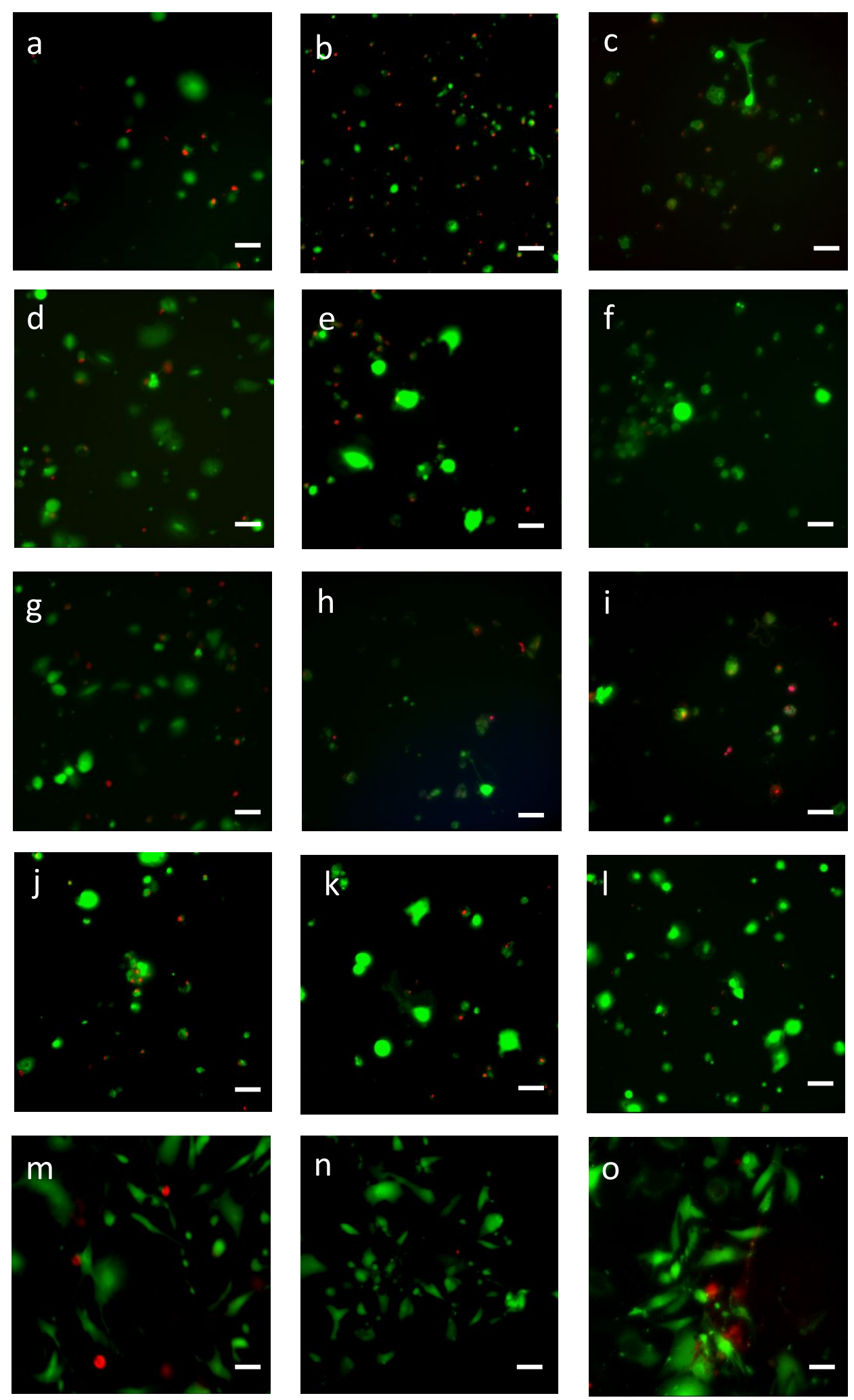

Figure S3. Comparison on the morphologies of hCSCs 3D and 2D growth at different time points. (a) - (c) 3D p(NIPAM-DMAEMA) microgel; (d) - (f) 3D p(NIPAM-PEGA) microgel; (g) - (i) 3D p(NIPAM-HEMA) microgel; (j) - (l) 3D p(NIPAM-IA) microgel; (m) (o) 2D culture. Scale bar is $20 \mu \mathrm{m}$. Green is Calcein (live cells), and Red is EthD (dead cells). 

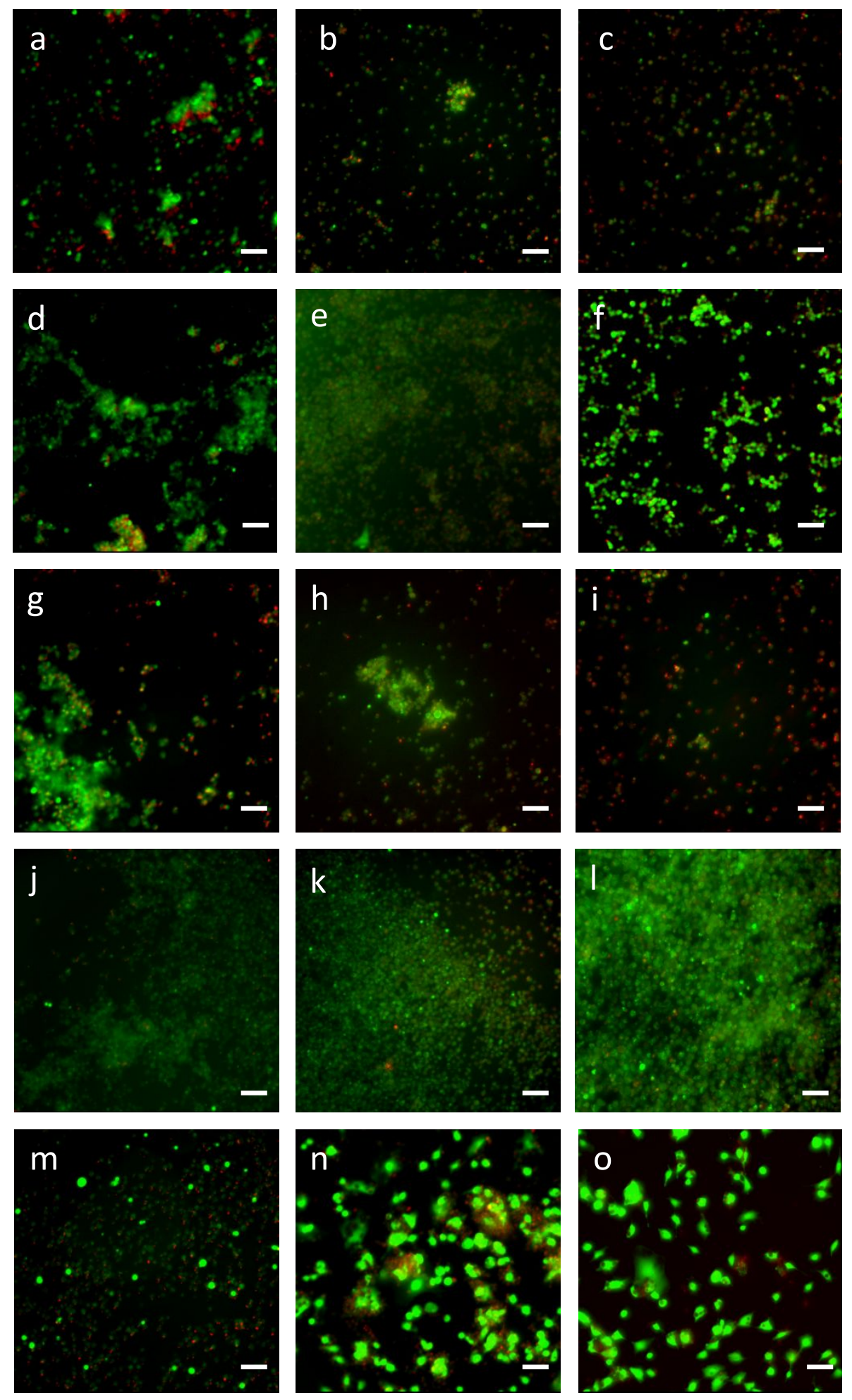

Figure S4. Comparison of the morphologies of NRCMs after 3D and 2D growth at different time points. (a) - (c) 3D p(NIPAM-DMAEMA) microgel; (d) - (f) 3D p(NIPAMPEGA) microgel; (g) - (i) 3D p(NIPAM-HEMA) microgel; (j) - (l) 3D p(NIPAM-IA) microgel; (m) - (o) 2D culture. Scale bar is $20 \mu \mathrm{m}$. Green is Calcein (live cells), and Red is EthD (dead cells). 